\title{
La possession du degré d’autonomie chez les vivants
}

\section{(The possession of degree of autonomy in living beings)}

\author{
PHILIPPE DALLEUR \\ Faculty of Philosophy \\ Pontifical University of the Holy Cross, Rome \\ dalleur@pusc.it
}

Résumé. Dans les nombreuses tentatives de définition du concept de vie, l'emploi de préfixes comme «self», «auto» apparaît de façon récurrente. Ce constat montre l'importance fondamentale accordée à l'autonomie chez les vivants. L'auteur se propose dans un premier temps d'analyser les divers types et degrés d'autonomie à partir de certains penseurs contemporains, comme Jonas, Morin, Varela, Davies, Wandschneider. Ensuite, les divers types d'autonomie systémique sont mis en parallèle avec les quatre niveaux de théories biologiques contemporaines (génétique, cytologie, cybernétique, évolution/ écologie). L'autonomie dépendante de la morphogenèse par rapport au niveau génétique est présentée comme un paradigme de l'autonomie systémique. Leémergence stratifiée et le confinement relatif des niveaux systémiques, ainsi que leurs interdépendances causales (upward et downward), révèlent une tendance vitale à augmenter et maximiser l'autonomie. L'étude des autonomies individuelles est alors présentée, et s'élabore autour des notions de feedbacks et de feed-forwards, d'homéostasie, d'autonomie motrice et trophique, pour se développer plus pleinement vers les autonomies émancipatrices et exploratrices, proprement autodéterminatrices. Ces derniers types d'autonomies supérieures résultent de propriétés, spécialement léquipotentialité liée à des attracteurs à bassins d'attraction plats étendus. Ces propriétés sont forgées par les niveaux inférieurs, en tant que logistique octroyant une capacité décisionnelle autonome au niveau supérieur. Lauteur suit la théorie de Jonas affirmant la tendance intrinsèque des systèmes vivants à produire des autonomies de plus en plus raffinées, dont le degré 
analogique suprême se trouve dans la liberté humaine. Ainsi l'autonomie est présentée comme un concept analogique applicable à tous les vivants, biologiques et spirituels.

Mots clés: autonomie ; liberté ; autopoièse ; biologie ; système ; attracteur ; complexité ; vie ; Jonas ; Thom ; autodétermination ; autocontrôle ; épigénétique ; individu ; émergence ; causalité descendante ; système à anticipation.

Abstract. In the numerous attempts to define the concept of life, the use of prefixes like "self", "auto" appears recurrently. This shows the fundamental importance attached to autonomy among the living beings. The author first analyzes the various types and degrees of autonomy, beginning from some contemporary thinkers, like Jonas, Morin, Varela, Davies, Wandschneider; and afterwards, the various types of systemic autonomy are compared with the four systemic levels of contemporary biological theories (genetics, cytology, cybernetics, evolution / ecology). The dependent autonomy of morphogenesis relative to the genetic level is presented as a paradigm of systemic autonomy. Stratified emergence and relative containment of systemic levels and their causal interdependencies (upward and downward), reveal life's tendency to increase and maximize autonomous levels. A study of individual autonomy is also presented and developed around the concepts of feedback and feed-forwards, homeostasis, motor and feeding autonomy, to develop more fully towards the emancipation and exploratory autonomies, as self-determinacies. These types of higher autonomies are the result of several properties, especially the equipotentiality related to attractors exhibiting flat spread basins of attraction. These properties are shaped by the lower functional levels as logistical granting for autonomous decision-making capability at the higher level. The author follows Jonas' theory affirming the intrinsic tendency of living systems to produce increasingly refined autonomies, whose supreme analog degree is found in human freedom. So, autonomy is presented as an analog concept applicable to all living, biological and spiritual.

Keywords: autonomy; freedom; autopoiesis; biology; system; attractor; complexity; life; Jonas; Thom; self-determination; self-control; epigenetic; individual; emergence; downward causation; feed-forward.

\section{Introduction : concept et « définitions » de la vie}

\section{Dans son Histoire de la notion de vie, André Pichot $(1993,5)$ affirme :}

Bien qu'elle nous touche de très près, la notion de vie n'a jamais été clairement définie, ni dans l'histoire des sciences ni dans celle de la philosophie. Sans doute parce qu'elle est difficile à saisir. D'elle on pourrait dire ce que saint Augustin disait du temps : «Qu'est-ce donc que la vie ? Si personne ne me le demande, je le sais; mais si on me le demande et que je veuille l'expliquer, je ne le sais plus.» (Augustin, IX, 14 ; Morin 1980, 349-350). 
De fait, la difficulté procède de la notion de vie elle-même, un concept fortement analogique et générique. La définition classique ajoute à un genre une différence spécifique infime. Par exemple on définit l'homme comme «animal rationnel». À une notion générique comme celle d'animal, on ajoute une différence spécifique propre à l'homme, sa rationalité. L'application d'une telle définition est relativement facile pour les concepts spécifiques, comme le cas des espèces vivantes. Mais plus le concept à définir est générique, plus il est difficile de lui trouver des différences vraiment spécifiques. Il en va ainsi du concept d'être, de vivant, de corps, de système, etc. La «définition» se réduit alors souvent à une liste de caractéristiques ou propriétés essentielles : une telle définition devient descriptive, et reste toujours à la merci de remises en questions de la liste des propriétés considérées comme essentielles.

Dans le cas de la notion de vie, les diverses «définitions» ont donné lieu à une pléthore de tentatives depuis l'Antiquité. Les anciens avaient une tendance à voir la vie comme irréductible aux mécanismes matériels, même si certains philosophes comme Démocrite et les atomistes, présentent une notion matérialiste de la vie ${ }^{1}$. Le vitalisme platonicien était proche des systèmes mythico-religieux de religions orientales et de l'animisme, avec sa croyance dans la chute des âmes dans les corps et la métempsychose. Dans le De anima, II, 1 et 2, Aristote définit le vivant comme source de fonctions ou d'activités caractéristiques qu'il énumère dans une liste plus descriptive qu'exhaustive : l'auto-alimentation, la croissance, la reproduction, le vieillissement et la mort, le mouvement et le repos (locomotion), la perception, l'intellection.

\section{L'autonomie des vivants}

Parmi les traits communs de ces descriptions diverses des vivants ou de la vie, on relèvera aisément l'apparition récurrente du préfixe «auto-» ou

Jacques Monod, dans son best-seller « Le Hasard et la Nécessité » (1973), cite en épigraphe une phrase de Démocrite ( t tout ce qui existe dans l'univers est le fruit du hasard et de la nécessité »), mais la citation est probablement apocryphe : quoiqu'elle reflète bien l'esprit de la physique atomiste de Démocrite, elle ne s’harmonise pas avec sa biologie.

ScientiaetFides 3(1)/2015 
«self-», et du suffixe «-même». ${ }^{2}$ Et dans cet échantillon de termes, il me semble que celui d'autonomie présente l'avantage de s'harmoniser analogiquement avec toutes les formes de vie que nous connaissons ou supposons, non seulement celles dues à la sélection naturelle, mais également d'autres formes de vie possibles, sans sélection naturelle ou sans reproduction, comme celles d'autres êtres de type matériel ou des esprits, qui ne sont pas ou plus soumis aux contraintes sélectives naturelles ${ }^{3}$. Tous ces êtres vivants, actuels ou potentiels, possèdent un certain degré d'autonomie. Le terme autonomie vient du grec auto, soi-même, et nomos, lois, signifiant par-là que l'autonomie est la propriété de suivre ses propres lois. Cette notion présente donc un degré d'aséité, d'auto-détermination.

En ce sens, se reposant la question de Schrödinger (1944), Paul Davies (2003, 9-10) écrit :

la propriété d'autonomie, ou autodétermination, semble toucher à l'aspect le plus énigmatique qui distingue le vivant des choses non vivantes, mais il est difficile de savoir d'où elle vient. Quelles propriétés physiques de l'organisme vivant leur confèrent une autonomie? Personne ne connait la réponse. L’autonomie est une caractéristique importante de la vie, mais il y en a beaucoup d'autres ${ }^{4}$.

L'autonomie n'est pas sans contraintes cependant, elle est toujours limitée d'une façon ou de l'autre. La théorie moderne des systèmes complexes a hérité des théories économiques et sociales des $18^{\mathrm{e}}$ et $19^{\mathrm{e}}$ siècles. Certains économistes du début du $19^{\mathrm{e}}$ siècle ont ainsi préparé la pensée évolutionniste et écosystémique en analysant l'évolution autorégulée de

2 Automotricité, auto-alimentation, autoreproduction, autoréplication, autorégulation, self-control, auto- ou self-assemblage, autoconservation, automatisme, autocatalyse, autonomie, autopoièse, self-existential unit, self-existence, self-creation, self-re-excitation, autoréparation, etc.

3 On pourrait aussi penser à des êtres ou espèces vivants, stériles par exemples, maintenus, obtenus ou même synthétisés par des techniques artificielles (OGM, microorganismes ou cellules comme vecteurs thérapeutiques, etc.).

4 La traduction est mienne. Davies cite aux pages suivantes 9 autres propriétés essentielles de la vie : reproduction, métabolisme, nutrition, complexité, organisation, croissance et développement, contenu informatif, interconnexion hardware/software, héritage permanent et variabilité. 
sociétés (ou systèmes sociaux) jouissant d'une certaine autonomie interne : par exemple l'économiste écossai initiateur du capitalisme, A. Smith, parle d'auto-développement des sociétés à concurrence libre; R. Malthus avec ses calculs prévoit les progressions ou régressions automatiques des populations en relation à l'accessibilité des ressources alimentaires ${ }^{5}$.

Le génie de Hans Jonas est de considérer que le moteur global de l'évolution est de progresser vers plus d'autonomie, dès les premiers niveaux de la vie, concrètement, le niveau métabolique. Il résume son projet dans l'introduction à son livre The Phenomenon of Life (Jonas 2001, ix) :

The great contradictions in which man discovers in himself - freedom and necessity, autonomy and dependence, self and world, relation and isolation, creativity and morality - have their rudimentary traces in even the most primitive forms of life, each precariously balanced between being and not-being, and each already endowed with an internal horizon of "transcendence." We shall pursue this underlying theme of all life in its development through the ascending order of organic powers and functions : metabolism, moving and desiring, sensing and perceiving, imagination, art, and mind - a progressive scale of freedom and peril, culminating in man, who may understand his uniqueness anew when he no longer sees himself in metaphysical isolation.

Pour Jonas, même les formes de vie les plus élémentaires préfigurent l'esprit, spécialement dans sa liberté. Ces formes possèdent en germe les plus hauts degrés de vie : des germes déjà actifs, mais de façon élémentaire. Le métabolisme est la marque de l'unité du vivant et c'est en lui que Jonas trouve ces germes universels du vivant. Le métabolisme permet une organisation indépendante et une continuité organique face à l'environnement, alors même que la matière est en flux continu (Jonas 2001, 75-76).

Ce métabolisme distingue et émancipe l'individu de l'environnement, tout en maintenant cette distinction tant que le corps est en vie. L'autonomie suppose l'individuation de l'organisme et la distinction du milieu

5 Wallace et surtout Darwin ont été influencés par la lecture de ses théories qui s'appliquaient non seulement aux sociétés humaines, mais encore aux sociétés animales et aux écosystèmes. 
extérieur. Pour la plupart des biologistes (Thom, Pichot, Varela,...) le bord de la forme vivante (peau, membrane, carapace, etc.) place une frontière, toujours connexe ${ }^{6}$, entre l'organisme, lieu d'organisation avec ses lois biologiques internes, et l'environnement, lieu anonyme des lois physicochimiques inanimées.

\section{Les niveaux opérationnels d'autonomies systémiques}

La notion d'autonomie s'applique à beaucoup de systèmes et conduit à des trames évolutives analogiques. Elle se retrouve à plusieurs niveaux dans les sciences de la vie, et pas uniquement en théorie évolutives. Hans Jonas, Francisco Varela et d'autres analysent une autonomie fondamentale des systèmes vivants. Des autonomies peuvent s'observer aux niveaux des classes d'organisation fonctionnelle et structurelle des vivants (genres, espèces, relations, écosystèmes, sociétés, etc.), mais aussi aux niveaux des instances individuelles, celles des objets vivants (individus, organes, etc.). Analysons maintenant le premier type générique d'autonomie de classe, ou autonomie systémique.

Comme le montre le tableau suivant, la biologie moderne s'est développée autour de quatre types de théories fondamentales, que l'on peut énumérer en partant du niveau le plus matériel et local (le niveau physico - moléculaire, le plus «fermé», le moins «autonome» pourrait-on dire), jusqu'au niveau le plus relationnel et global (le niveau écosystémique de la biosphère, le plus «ouvert» et pas nécessairement le plus autonome). Ces quatre types de disciplines couvrent $: 1^{\circ}$ la génétique $; 2^{\circ}$ la cytologie ou théorie de la cellule ; $3^{\circ}$ la cybernétique et l'étude des systèmes complexes; $4^{\circ}$ l'évolution et l'écologie.

\footnotetext{
La connexité exprime que l'organisme individuel est enfermé dans un espace contigu, aussi difforme qu'il peut être, et non dans des espaces séparés. La question se pose de la possibilité d'une contiguïté énergétique ou informatique, avec discontinuité spatiotemporelle : certains assimilent ainsi à un seul «hyper-organisme» les colonies de fourmis, les sociétés ou même toute la biosphère (comme dans le cas de certaines interprétations de l'hypothèse Gaia). De toute façon, le «soi» doit être séparé des autres et de l'environnement (Varela 1997, 75).
} 


\begin{tabular}{|c|c|c|}
\hline Théorie (*) & Niveaux de complexité ( $\left.{ }^{* *}\right)$ & Causalité \\
\hline I. Génétique (5) & $\begin{array}{l}\text { Physico - moléculaire : lois physiques, } \\
\text { macromolécules (protéines, ARN, } \\
\text { ADN, polymères), information de base, } \\
\text { héritage, etc. }\end{array}$ & \multirow[t]{2}{*}{ + matérielle } \\
\hline II. Cytologie (1) & $\begin{array}{l}\text { Cellulaire - vital de base : organelles, } \\
\text { membranes, noyau, osmose, etc. }\end{array}$ & \\
\hline III. Systèmes (3) & $\begin{array}{l}\text { Organique - individuel : homéostasie, } \\
\text { autopoièse, régulations, feed-backs, } \\
\text { feed-forward, intégration, réparation, } \\
\text { croissance, reproduction, morphogenèse, } \\
\text { émergences, etc. }\end{array}$ & \multirow[t]{2}{*}{+ formelle } \\
\hline $\begin{array}{l}\text { IV. Évolution (2), } \\
\text { écologie (4) }\end{array}$ & $\begin{array}{l}\text { spécifique - écologique : adaptations avec } \\
\text { causalité top-down (héritage génétique), } \\
\text { exaptations, écosystème, biosphère, etc. }\end{array}$ & \\
\hline
\end{tabular}

(*) Les chiffres entre parenthèses expriment l'ordre chronologique de l'apparition de ces théories : la théorie cellulaire est historiquement la première à ouvrir la porte à la biologie moderne, grâce à l'invention du microscope optique.

(**) La complexité croît vers le bas. On passe aussi du niveau le plus matériel au niveau le plus formel.

Aux quatre niveaux fondamentaux dans l'organisation des vivants correspondent des niveaux ou degrés de structures différentes, de matérialité décroissante et de complexité croissante. L'autonomie «organisationnelle» interne se divise en deux types d'autonomie. Les agents, leurs lois (rappelons-nous que nomos signifie loi en grec) et leurs propriétés actives diffèrent entre les niveaux, ce qui octroie d'un côté une certaine autonomie structurelle entre les niveaux, mais aussi une autonomie fonctionnelle. Souvent structure et fonctions sont interdépendantes : la structure agence les fonctions qui maintiennent ou renforcent la structure.

Mais les «lois» biologiques de chaque niveau diffèrent : la division cellulaire suit une cascade d'événements très différents des parades nuptiales des vertébrés supérieurs ; notre comportement social ignore quasi 
totalement les dynamiques intracellulaires de nos cellules. Les quatre niveaux cités sont suffisamment marqués pour conduire à quatre types de disciplines ou théories biologiques fondamentales, chacune spécialisée à l'étude d'aspects particuliers du vivant. Les deux premières (génétique et cytologie) sont plus tournées vers l'analyse des mécanismes élémentaires ou microscopiques, et constituent le niveau de base, plus matériel parce que plus proche des sciences exactes, essentiellement la chimie et la physique, thermodynamique comprise. Les deux suivantes (la cybernétique et l'évolution) sont plus associées à une vision holistique, phénoménologique, morphogénétique, écosystémique et sociale.

Ces types d'autonomies stratifiées en niveaux sont du même type que l'autonomie métabolique citée par Hans Jonas comme base commune des vivants, dans le sens où ces autonomies internes s'assimilent à des isolements opératifs. Le bord du corps isole ce dernier, le distingue de l'environnement, et délimite le lieu où s'exerce le contrôle métabolique. Le métabolisme contrôle l'organisme vivant jusqu'à son bord, et s'arrête là. Il contrôle le corps circonscrit dans sa peau ou sa membrane. Cela n'empêche pas le métabolisme de « déborder » du corps au niveau du bord pour des échanges énergétiques et les rejets toxiques. De même, les individus d'une même espèce s'organisent pour la survie de l'espèce, selon une organisation essentiellement interne à l'espèce, « débordant » aussi pour l'accès aux ressources ou la défense contre des prédateurs par exemple.

L’autonomie s'appuie sur des dépendances. Des sociologues comme Edgar Morin et Robin Fortin ont étudiés le concept d'autonomie dans les systèmes sociaux complexes. Comme Hans Jonas, Morin estime que l'autonomie présuppose une dépendance énergétique par rapport à l'environnement. Morin développe aussi l'idée que l'autonomie croît avec la dépendance (Morin 1980, 148 ; Fortin 2005, 63) Par exemple, le téléphone portable nous offre plus de possibilités et d'autonomie qu'auparavant (au niveau supérieur de communication et de travail), nous sommes cependant plus dépendants de la technologie (au niveau inférieur, celui du matériel technologique). J'ajouterai que la plus grande autonomie du niveau supérieur (vivant, humain) est une autonomie formelle assurée par une dépen- 
dance matérielle aux niveaux structurels inférieurs (inanimés, végétatifs, etc.). Ainsi apparaît une structure verticale de niveaux qui s'empilent l'un par-dessus l'autre, un peu à la manière des plantes verticillées, qui ont des ramifications par paliers (Dalleur 2008, 79-83) ${ }^{7}$.

Cette dépendance est non seulement externe, mais aussi interne, vu que les processus élémentaires de base se fondent sur le fonctionnement matériel avec ses lois physico-chimiques. Le mathématicien et biologiste français René Thom considère que la théorie des systèmes rend mieux compte de la morphogenèse que les théories évolutionnistes. Les formes vivantes possèdent des lois propres relativement indépendantes de la matière et de la mécanique physico-chimique sous-jacente associées à elle. Les cellules, les organes ou les individus fonctionnent comme des boites noires (black boxes) ou des modules fonctionnels. Au niveau fonctionnel supérieur, peu importe la mécanique interne, pourvu qu'elle fonctionne correctement. Ce type d'autonomie qui distingue l'intérieur caché de la boîte noire à son interface visible, phénoménologique, est comparable aux appareils comme par exemple les horloges. Certaines horloges sont purement mécaniques, d'autres sont purement électroniques, d'autres encore électriques : cela correspond à leur fonctionnement interne caché à l'utilisateur. Mais leur « fonction sociale » est équivalente, car elle peut être remplie indifféremment par toutes les horloges fonctionnelles : elles donnent toutes la même heure pour que nous puissions régler nos vies et nos rendez-vous. Certaines consomment moins d'énergie, sont plus visibles, de couleur différente, etc. mais tous ces détails de « cuisine interne » importent peu, pourvu que l'horloge donne l'heure.

Un signe de cette autonomie s'observe dans l'équivalence fonctionnelle au niveau supérieur entre diverses entités, dont les fonctionnements internes du niveau inférieur diffèrent grandement. Chacune de ces entités fonctionne comme des modules (ou boites noires), équivalents entre eux extérieurement et phénoménologiquement, parfois interchangeables au niveau supérieur. L'interface au niveau du bord de la forme est ce qui

7 Cet article m’a servi à l'élaboration du présent article. 
compte pour le niveau supérieur, c'est-à-dire comment la boite noire réagit avec l'environnement externe et s'intègre à lui. Il importe de voir ici qu'il existe une grande diversité de réalisations internes possibles de boites noires biologiques offrant la même fonctionnalité. En sciences de la vie, l'équivalence fonctionnelle aux niveaux supérieurs s'observe dans les convergences évolutives, les greffes, les mosaïques cellulaires, etc. Par exemple, le loup de Tasmanie, avant sa décimation par l'homme, occupait une niche écologique équivalente au loup ou au renard. Il est remarquable de voir comment ce marsupial australien non placentaire, très éloigné des canidés eurasiatiques aux niveaux génétique et phylogénétique (les niveaux inférieurs), a acquis une forme phénoménologique prédatrice (le niveau supérieur ; comportement, dentition, silhouette, museau, odorat, oreilles, queue, pattes, squelette, etc.) quasi identique à celle des canidés placentaires. Pour Thom, la prédation est le «moteur» morphogénétique le plus puissant, la raison première de l'apparition des formes vivantes et de leur morphologie. Il estime que les explications en termes de «sélection naturelle» et «d'adaptation à l'environnement» sont trop génériques et masquent la dynamique profonde de la vie, sans pouvoir rendre compte de la diversité des espèces dans un même environnement.

Les oiseaux, les insectes volants, les chauves-souris, ont acquis des ailes pour voler, même si la réalisation de celles-ci diffère énormément par les gènes activés et les croissances tissulaires. Leurs ailes sont comme des « prédations » (au sens analogique) sur l'air ambiant. Le principe fondamental et premier est que le «prédateur » (ou ce qui s'assimile morphogénétiquement à un prédateur) s'adapte à ses proies ${ }^{8}$. Les proies ne sont pas nécessairement vivantes. Elles sont même fréquemment inanimées, comme l'air respiré, l'eau absorbée, ou même les données sensibles mémorisées. La fractalisation des organes est la réponse de la vie à ces échanges microscopiques, nutritifs, respiratoires ou informationnels : on l'observe dans la ramification des bronches, des vaisseaux sanguins, des nerfs et des dendrites, des plis intestinaux, des papilles et des lobes cérébraux. En fin

8 On pourrait en dire de même de l'analogie entre les nageoires des poissons et celles des mammifères marins. 
de compte peu importe la génétique sous-jacente, pourvu que celle-ci fonctionne correctement au niveau inférieur, comme « cuisine interne ». Ainsi, si la vie apparaissait indépendamment sur une autre planète semblable à la terre, elle exhiberait des formes des systèmes nutritifs, respiratoires et informatifs ... similaires, bien que leur génétique de base puisse être totalement différente et même incompatible avec le système de codification terrestre de l'ADN.

Chaque niveau se caractérise par une certaine autonomie opérationnelle dérivant des différences d'organisation, de structure, de lois et d'agents fonctionnels. La croissance de la complexité est liée à l'interaction de parties plus simples et élémentaires. Ce schéma d'organisation opérationnelle correspond curieusement à la méthode analytique de Descartes (dans son Discours de la méthode) : pour comprendre ou étudier le niveau supérieur, il faut le découper en parties plus simples à étudier, en espérant pouvoir comprendre le tout par l'étude des parties. En réalité la théorie des systèmes montre qu'il est souvent impossible de réduire complètement un niveau supérieur au niveau inférieur de ses parties, en particulier dans les systèmes proprement complexes. Il existe des phénomènes émergents, irréductibles aux niveaux inférieurs. Les lois de fonctionnement du niveau supérieur se basent sur celles des niveaux inférieurs (génétiques, physiques, chimiques). Cependant il est important de noter que les niveaux inférieurs, loin de contraindre le fonctionnement du niveau supérieur, lui octroie une certaine «liberté» fonctionnelle, pour emprunter le terme utilisé par Hans Jonas 9 .

Le tableau de classification des théories peut donner l'impression que les disciplines et niveaux sont relativement indépendants. Les théories de l'évolution par sélection naturelle affirment cependant que les adaptations à l'environnement du niveau supérieur conduisent à une causalité descendante (de type top-down, appelée en anglais downward causation) qui sélectionne la génétique plus favorable ou efficace. Ainsi les niveaux

9 Pour ma part, je préfère parler d'autonomie formelle au niveau supérieur, et restreindre l'utilisation du terme «liberté» à un autre niveau, le rationnel, proprement réservé à l'homme. 
se complètent et coopèrent, ce qui a pour effet d'optimiser leur efficacité globale. Celle-ci est essentiellement liée à d'autres types d'autonomies opérationnelles, plus externes. Mais en réalité chaque niveau est en interaction étroite avec les niveaux voisins. Le monde vivant est un véritable laboratoire cybernétique où abondent à tous les niveaux des rétro-alimentations, plus connues sous le vocale anglo-saxon de feedbacks, des structures fonctionnelles universelles, du moins en biologie. Ainsi, l'étude interne des cellules en cytologie ne peut pas faire abstraction de la théorie des systèmes. Les feedbacks (ou bouclages régulateurs à réaction positive ou négative) dominent les interactions intracellulaires. Aux autres niveaux, l'évolution, l'ontogenèse, les épidémies, les durillons, le renforcement synaptique, la multiplication cellulaire, la vie elle-même, ... sont grossièrement assimilables à des systèmes à feedbacks positifs.

\section{Les autonomies individuelles internes}

Jusqu'ici nous avons analysé les autonomies de classe, dont l'archétype a été l'autonomie morphogénétique par rapport à la génétique, c'est-à-dire une autonomie fonctionnelle générique et non pas une autonomie proprement individuelle. Au niveau individuel, on retrouve plusieurs mécanismes cybernétiques similaires : des feedbacks et des feed-forwards entrent en jeu pour donner au vivant concret une certaine autonomie de fonctionnement, une certaine « liberté » d'action pour emprunter la terminologie de Jonas, ou une autopoièse $e^{10}$ pour reprendre le néologisme inventé par Francisco Varela et Umberto Maturana. Ce type d'autonomie se présente davantage comme une auto-possession accrue de son propre être et agir. Comme auto-possession, elle préfigure l'autonomie de la liberté des êtres spirituels.

Les feedbacks négatifs tendent à éliminer toute perturbation du système. Ils servent en particulier à maintenir l'homéostasie, la tendance à conserver l'état interne le plus proche possible de l'état «standard » de

$\overline{10}$ De auto, soi-même, et poiesis, production, réalisation. 
vie. Ces contrôles permettent de s'affranchir de dérives mortelles et manifestent une certaine autonomie conservatrice (type d'autonomie fonctionnelle essentiellement individuelle, mais parfois de groupe comme dans les cas de coopérations ou de symbioses) dont le paradigme est l'homéostasie. Pensons à la régulation de la température chez les oiseaux et les mammifères. Un feedback utilise une évaluation de la température et déclenche une série de réactions internes (tremblements, sueur, etc.) destinés à maintenir la température idéale. Les reptiles n’ont pas de régulation interne et doivent parer à ce manque en s'exposant à l'ombre et au soleil selon le cas. Cette organisation interne ne leur permet pas de vivre dans les régions polaires, trop froides pour maintenir une activité vitale non régulée. Le métabolisme d'un mammifère isole davantage son organisation interne des conditions externes.

\section{Feed-forwards et autonomies externes : motrices, énergétiques, trophiques}

A plus d'autonomie fonctionnelle correspondent souvent plus d'autonomies externes : autonomie motrice, énergétique (par exemple endurance, migrations), etc. Au niveau individuel, l'autonomie motrice des plantes est généralement plus restreinte que celle des animaux, mais pas leur autonomie trophique. Les plantes autotrophes produisent tous les acides aminés dont elles ont besoin : ce n'est pas le cas des animaux, hétérotrophes, qui dépendent des plantes à ce niveau. Ainsi, les plantes ont pu conquérir les continents avant que les animaux terrestres ne s'y aventurent.

Les contrôles des feedbacks présentent un inconvénient qui limite leur efficacité et leur capacité à l'optimisation parfaite : ils ne sont pas instantanés! Ils exhibent un délai de rétroaction sortie-réentrée, lors de la rétro-alimentation des paramètres structurels à contrôler. Ce délai peut même rendre le contrôle difficile voire impossible à réaliser en cas d'urgence. Heureusement, il existe aussi des systèmes à anticipation, véritable panacée biologique, baptisés en anglais feed-forwards, capables de prévoir la réaction future du système et à partir de là en principe, d'éliminer tout 
délai de réaction et annuler toute déviation non optimale. Cela dépend bien sûr de la capacité prédictive et de la rapidité du système anticipateur, ce qui suppose souvent une modélisation interne des fonctions et des réactivités dynamiques du système, qui doit toujours posséder des caractéristiques ou lois stables sur lesquelles le système anticipateur peut appuyer sa prédiction. Le feed-forward simple est à « boucle ouverte » (open loop, c'est-à-dire sans rétroaction sortie-entrée). Mais, en pratique les feed-forwards se combinent presque toujours à l'un ou l'autre feedback. Pour illustrer la différence, disons qu'un feedback ressemble à la poursuite d'un fugitif : si ce dernier tourne à droite, le poursuivant tournera aussi à droite, quoiqu'un peu plus tard. Par contre, le feed-forward correspondrait par exemple à l'attente du fugitif à l'endroit où il doit passer, ou au contrôle de sa fuite (par variations d'itinéraires, d'obstacles, ...) pour le faire aller vers un point de capture. Le sens de la vue octroie un avantage capital : elle permet à l'individu de vivre presque constamment plongé dans un univers de feed-forwar$d s$, pour éviter des obstacles ou des prédateurs, chercher efficacement ses proies, protéger sa progéniture, échapper à des accidents, etc.

Les feed-forwards donnent au vivant une série d'autonomies supplémentaires, typiquement une certaine indépendance par rapport au temps, une autonomie temporelle, quand ils anticipent des problèmes futurs pour les éliminer avant qu'ils n'arrivent. Les exemples abondent, depuis les migrations qui permettent aux oiseaux de s'échapper des conditions climatiques mortelles avant de les souffrir, jusqu'à la morphogenèse des poumons chez les fœtus, qui leur permettront de survivre hors du sein maternel après leur naissance, en passant par la perte des feuilles, l'hibernation ou l'emmagasinement de miel pour passer l'hiver. L'espèce survit grâce à des mesures anticipatives, et non pas conséquentes comme dans le cas des feedbacks.

Au niveau de l'individu, pratiquement toutes les adaptations résultent en fin de compte de feed-forwards qui pré-adaptent l'individu grâce à une mémoire spécifique héritée qui s'est affinée par l'histoire de la sélection naturelle. L'évolution n'agit pas directement au niveau de l'individu vivant (ce qui serait alors plus lamarckien que darwinien), mais au niveau de l'his- 
toire de la sélection des individus engendrés, plus comme une élimination de l'inapte (et donc agissant directement sur l'infertilité ou la mort des inaptes) qu'une "survie du plus apte ». De plus, le pool des vivants «moins aptes » est souvent immensément plus nombreux et actif que «le plus apte ", au point de pouvoir statistiquement produire une progéniture plus apte que les plus aptes actuels.

Combinés à l'autonomie motrice, les feed-forwards perfectionnent la prédation : le prédateur peut optimiser ses stratégies, affiner son habilité et son astuce pour augmenter ses chances d'attraper ses proies. Pensons aux toiles d'araignées, aux stratégies des loups, des félins, des serpents, etc. De leur côté, les proies affinent leurs fuites ou stratégies pour augmenter leurs chances d'échapper aux prédateurs. Remarquons que ces ajustements et affinements ne sont donnés par l'espèce (la nature, l'essence) à l'individu que comme potentialité : une potentialité qu'il s'agit pour le vivant de développer individuellement par répétitions d'actes, d'entrainements éducatifs, d'imitation des adultes, etc. Certains comportements ne sont pas complètement innés, mais il existe, surtout chez les animaux supérieurs, une part plus ou moins importante d'acquis individuel. Dans une démarche proche de Jonas - qui estime que l'autonomie des vivants préfigure la liberté humaine -, nous pourrions dire que cette acquisition d'habilités par répétition d'actes, préfigure l'exercice des vertus et des habitus opératifs moraux chez l'homme.

\section{Les autonomies émancipatrices et exploratrices.}

Jusqu'ici nous avons évoqué des types d'autonomies opérationnelles adaptées à un contrôle accru d'un domaine d'organisation, une sorte « d'autocontrôle ». Mais il existe aussi un autre aspect de l'autonomie qui préfigure un autre aspect de la liberté humaine, celui de l'absence ou la libération de contraintes. Ce nouvel aspect est parallèle et conjoint aux autonomies organisatrices que nous avons étudiées. Il ne s'agit pas seulement d'avoir un contrôle sur soi ou sur autrui, mais encore pour avoir un véritable contrôle, de ne pas être contrôlé par un autre sous le même rapport («l'autre » peut 
être un individu, l'environnement ou un niveau d'organisation). Les autonomies dont nous avons parlé, se libèrent toutes dans une mesure plus ou moins grande, de l'action de l'environnement sur le corps vivant ou sur l'espèce, etc. C'est une condition pour pouvoir avoir un contrôle efficace. L'évolution va dans le sens de l'efficacité du contrôle de soi et donc indirectement sur l'absence du contrôle par autrui (ou au moins le contrôle du contrôle par autrui).

La vie tend à produire plus d'autonomie, de liberté, à s'émanciper en partie de contraintes matérielles inférieures. Nos gènes ne déterminent pas le type de métier ou le travail que nous réalisons, mais ils nous octroient une base somatique pour un espace de liberté nous permettant d'agir et de travailler comme nous l'entendons. Si la génétique s'arrête, nous ne pouvons plus travailler, mais si elle fonctionne elle nous laisse le choix du travail et de nos actions. Hans Jonas va plus loin encore et entend donner une vision intégrée du vivant qui répare la rupture du dualisme cartésien et lui permette d'établir un pont entre le niveau biologique du métabolisme et le niveau supérieur réservé à l'homme, le niveau rationnel et éthique où la liberté n'est plus une simple autonomie biologique ${ }^{11}$.

Pour Dieter Wandschneider (2005, 196-215), l'organisation des vivants se structure dans cette dimension, celle de la croissance de l'autonomie. Nous savons que les plantes autotrophes produisent tous les acides aminés des vivants et libèrent de l'oxygène. Cela permet aux animaux de se développer et de vivre en profitant de leurs supports logistiques. La nature utilise une sorte de division du travail. L'animal est ainsi libéré de certaines contraintes matérielles pour être plus agile, plus efficace. À un niveau supérieur, les carnivores profitent de la difficile digestion des plantes par les herbivores pour disposer de sources énergétiques et protéiques concentrées. Ici aussi on observe un empilement de niveaux d'autonomies émancipatrices avec dépendance logistique accrue, qui al-

11 "A new reading of the biological record may recover the inner dimension - that which we know best - for the understanding of things organic, and so reclaim for the psychophysical unity of life, that place in the theoretical scheme which it had lost through the divorce of the material and mental since Descartes." (Jonas 2001, xxiii) 
lègent et simplifient le métabolisme de l'animal supérieur. Ce support découle des synergies entre vivants. L’animal supérieur peut alors simplifier sa complexité de base et spécialiser sa complexité supérieure dans des domaines différents ou nouveaux, qu'il serait impossible d'explorer sans ce support logistique. L'évolution apparaît ainsi comme stratifiée. A chaque niveau il y a une évolution horizontale qui explore les possibilités internes du niveau : par exemple, les plantes évoluent à leur niveau sans nouveauté émergente. Mais en même temps, elles induisent des effets structurels sur l'environnement (rejets d'oxygène, capture de $\mathrm{CO}_{2}$, matériels organiques, etc.) qui permet l'apparition d'un niveau émergeant différent des plantes, celui des animaux. Les endo- ou bio-symbioses et les sauts évolutifs (étudiées par Margulis, Gould, Conway Morris) complètent ce schéma évolutif vertical, qui résulte souvent de catastrophes environnementales globales (chocs de météores gigantesques, fortes glaciations, réchauffements généraux, volcanisme très actif, etc.). Celles-ci ont accru énormément la pression sélective sur les quelques survivants. Suite à ces accidents, de nouvelles formes de vie apparurent avec des changements génétiques, synergétiques ou comportementaux importants. Cela s'est concrétisé dans l'apparition subite de niveaux morphogénétiques autonomes supérieurs, avec une dépendance logistique accrue envers les niveaux inférieurs. Les variations de l'environnement ou l'influence environnementale des organismes inférieurs peuvent contribuer à des changements d'adaptation, à l'inutilité d'adaptations précédemment utiles, à certaines exaptations ${ }^{12}$ qui ouvrent de nouvelles possibilités avec réduction du prix énergétique ou de l'effort à fournir, donc avec simplification des complexités trop lourdes à porter.

Dans le même sens, Thom fait remarquer que l'ouverture potentielle d'un nouveau niveau n'est pas nécessairement liée à un accroissement de

12 Les exaptations sont des effets collatéraux d'adaptations préalables et pour des fonctions indépendantes. Ainsi, certains estiment que les plumes et les ailes des oiseaux sont initialement apparues suites aux pressions adaptatives liées à l'isolation thermique et non au vol. L'adaptation au vol s'est développée ensuite comme évolution postérieure à partir d'un avantage collatéral chez certaines espèces arboricoles, pour échapper aux prédateurs par un saut suivi d'une chute amortie par l'extension des plumes. 
contraintes morphogénétiques (et donc aussi de gènes et de dimensions des attracteurs), mais qu'une réduction de dimension d'un attracteur peut conduire à une libération, une croissance accrue ou une fractalisation d'un tissus (Dalleur 2006, 319 ; Thom 1980, 262). En morphogenèse, la perte d'une contrainte entraine parfois un avantage en termes de degrés de liberté. De façon imagée, je pourrais dire qu'une table a trois pieds est assurément plus stable qu'une à deux pieds, mais aussi que cette dernière a plus de possibilités de mouvements que la première qui est fixée dans son état. Pour qu'un navire aille au large et explore les océans, il faut lâcher ses amarres. La croissance de l'autonomie n'est pas nécessairement parallèle à la croissance de la complexité. L'évolution n'est pas simpliciter synonyme de complexification, ainsi que Darwin lui-même l'avait remarqué dans l'analyse des formes ataviques atrophiées et des régressions évolutives ${ }^{13}$. Plusieurs théoriciens des systèmes contemporains estiment que la simplification ou le relâchement de contraintes peut permettre plus d'exploration que l'accroissement de complexité ou de contrôle. Comme le montre Francis Heylighen, le contrôle d'un paramètre restreint son autonomie, le relâchement du contrôle l'augmente (Heylighen 2008, 1220). De même, le relâchement de certaines protections complexes d'une série de gènes contre les mutations, permet une mutabilité accrue et donc une évolution (ou «évoluabilité») accélérée de ces gènes, ce qui peut être intéressant dans certains cas où une espèce doit s'adapter rapidement à des changements environnementaux ou des prédateurs variables. La vie utilise toute une série de contrôles épigénétiques qui activent ou inhibent l'expression et la mutabilité génétique (méthylation, replis, chaperons, etc.). On parle de contrôle (épigénétique) du contrôle (génétique). Le réseau de l'organisme est ainsi constitué de niveaux relativement indépendants, et qui cependant peuvent moduler les autres niveaux suivant des causalités downward ou upward.

13 En ce sens, Edgar Morin prévient aussi du danger de confondre aisément complexité et complication. 


\section{L’autonomie équipotentielle des attracteurs tabula rasa et omnia fieri.}

La nature offre aussi des niveaux supérieurs d'autonomie équipotentielle. Ce type d'autonomie est gratifié par le niveau inférieur au niveau supérieur, en tant qu'ouverture, parfois illimitée, du champ des possibilités («open-ended field of possibilities»), sans être capable de déterminer l'agir fonctionnel de ce dernier. Le niveau inférieur prépare pour le niveau supérieur un support ou une base «neutre», comparable au «tamquam tabula rasa» des scholastiques. Il fait idéalement «table rase» des influences biaisées, des contraintes univoques, pour laisser la voie libre aux décisions ou actions du niveau supérieur. Le niveau inférieur offre un contenant ou une base neutre, garantit sa neutralité ou même son incapacité décisionnelle et fonctionnelle, laissant au niveau supérieur le soin du choix du contenu, idéalement sans l'influencer. Ainsi en va-t-il normalement de toute logistique : celle-ci est nécessaire pour allouer un environnement, idéalement sans (pré)contraintes. Le niveau supérieur dépend de l'inférieur en tant que celui-ci donne les conditions nécessaires pour une autonomie décisionnelle ou fonctionnelle, sans donner de condition suffisante. Le niveau inférieur ne fait qu'apporter un support matériel et logistique à la décision.

Cette autonomie supérieure n'est possible que si le système inférieur est capable de se placer (ou d'être placé) dans un état neutre, indifférent et indéterminé, qui autorise plusieurs évolutions futures différentes (voire même une infinité de possibilités), impossibles à prédéterminer à son niveau. Seul le niveau supérieur pourra déterminer la voie suivie. On peut reprendre graphiquement l'image du paysage épigénétique chère à Waddington. Dans la figure ci-jointe, la bille $\boldsymbol{a}$ représente l'état du système de paramètres morphogénétiques $\mathrm{x}$ et $\mathrm{y}$, dans un attracteur ${ }^{14} \mathrm{~A}$, contrairement à la bille $\boldsymbol{b}$ dont les paramètres $\mathrm{x}$, $\mathrm{y}$ évoluent au point de bifurcation $\mathrm{B}$, selon

14 Toute auto-organisation suppose que le système évolue jusqu'à atteindre un attracteur, c'est-à-dire, une partie de l'espace d'état accessible et où l'état est aisément capturé de façon relativement stable (comparable à l'équilibre stable qui revient vers l'attracteur lorsqu'il s'en est éloigné au sein du bassin d'attraction). 


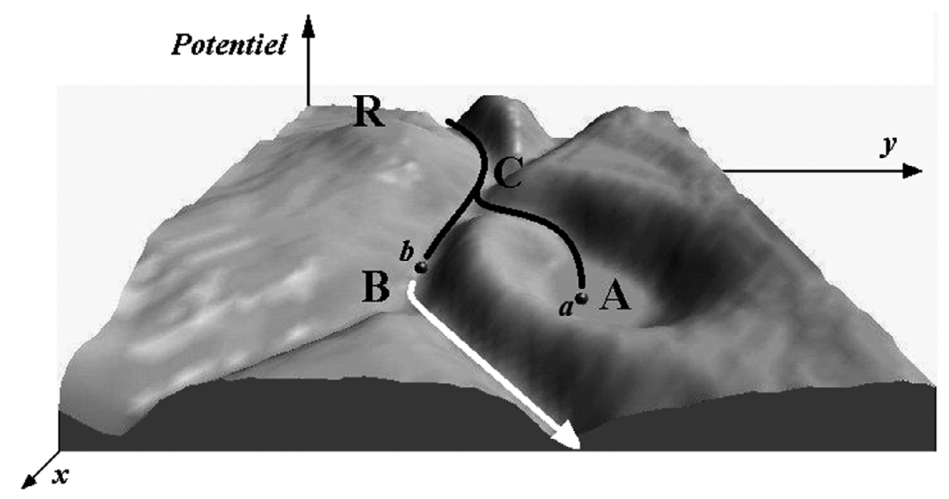

une trajectoire évitant le point de rebroussement $\mathrm{R}$ et l'attracteur A. L'état $\boldsymbol{a}$ se trouve prisonnier de cet attracteur qui montre ici une forme spéciale : le fond de son bassin d'attraction est plat, tamquam tabula rasa. L'attracteur n'est pas ponctuel, mais est dégénéré en une zone équipotentielle, qui offre une infinité d'états possibles (le omnia fieri potest) dans le bassin d'attraction pour le même niveau potentiel. La position finale de l'état $\boldsymbol{a}$ est imprédictible ainsi que son évolution. L'évolution du niveau supérieur est irréductible aux niveaux inférieurs, qui sont réglés pour produire et dégénérer certains attracteurs.

Dynamisme ponctuel

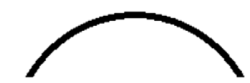

Rebroussement ponctuel

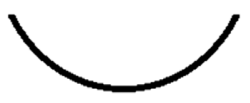

Attracteur ponctuel
Equipotentialité

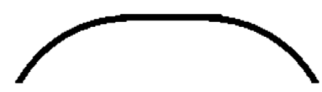

Rebroussement plat

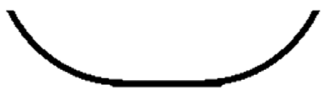

Attracteur plat 
La nature produit beaucoup d'événements «ouverts» associés à des états systémiques équipotentiels, en ce sens que leurs niveaux inférieurs de fonctionnement ne déterminent pas univoquement l'évolution future du système au niveau supérieur, mais qu'ils offrent un espace ouvert d'autonomie évolutive, de développement a priori indéterminé, imprédictible. Les puissances opératives naturelles déterminent généralement l'agir concret, qui correspondra à un attracteur ponctuel. L'instinct animal permet de résoudre des cas précis : l'abeille danse en 8 , le chien enterre sa nourriture, etc. Cependant, lorsque le champ des possibilités est vaste et complexe, il peut être préférable de ne pas donner une solution toute faite, pré-câblée, aux divers problèmes possibles, et de doter le système d'une ample autonomie, avec un potentiel élevé de possibilités équiprobables (omni-et équi-potentialité), jointe à une capacité de détermination ad casum. L'auto-déterminabilité cybernétique imite celle des animaux supérieurs et peut nous aider à comprendre ce fait. Les premières sondes martiennes étaient fixes au sol. Elles étaient beaucoup moins efficaces que la nouvelle génération de «sondes exploratrices» nettement plus «autonomes», comme la sonde robotique Curiosity qui s'est posée automatiquement et évolue assez indépendamment sur le sol martien depuis août 2012. L'autonomie ou la déterminabilité ${ }^{15}$ est une propriété essentielle des organismes supérieurs pour explorer efficacement les espaces de possibilités potentiellement infinies ouvertes à une puissance active. Ce que ne représentent pas les figures ci-dessus est la décision et l'action autonome supérieure qui détermine l'état $\boldsymbol{a}$ dans le bassin d'attraction.

Par exemple, le tabula rasa et l'équipotentialité initiaux des sens, des puissances cognitives et opératives, n'est pas une simple indétermination, mais une déterminabilité, une capacité prête à lever l'indétermination. Nous utilisons quantité d'objets qui sont produits avec un certain niveau

15 Comme Paul Davies, cité plus haut, je préfère employer le terme de déterminabilité à celui d'autonomie, qui rend compte génériquement de l'auto-déterminabilité du niveau supérieur, mais celle-ci peut être auto-déterminabilité individuelle, ou déterminabilité «ab alio» au sein même du niveau supérieur, comme dans le cas d'apprentissage, d'éducation, d'empathie, d'imitation. 
d'équipotentialité. Par exemple, un cahier composé de pages blanches sert de support matériel à l'écriture de n'importe quel message; un écran de projection est blanc parce qu'il peut recevoir n'importe quelle image ; le clavier d'un ordinateur permet d'écrire n'importe quel texte. De même chez les vivants, la polymérisation des bases ACGT de l'ADN sert potentiellement à la synthèse de n'importe quelle protéine; la transparence de l'humeur vitrée et du cristallin et le tapissage cellulaire de la rétine captent n'importe quelle image ; la locomotion permet un déplacement sur le sol dans n'importe quelle direction. Ces autodéterminations ad casum octroient au vivant une capacité de réaction aux imprévus.

Les principes systémiques associés du tamquam tabula rasa et de l'omnia fieri $^{16}$ se rencontrent aussi dans les phénomènes d'émergence de niveaux de complexité. Lorsqu'un système évolue, de nouvelles complexités peuvent émerger en son sein, irréductibles aux complexités antérieures. Elles jouissent alors d'une certaine autonomie, sans quoi il n'y aurait pas d'émergence : le type de complexité change dans cette émergence supérieure.

\section{Conclusions}

Le concept d'autonomie semble être l'un des plus féconds pour expliquer le vivant, ainsi que l'ont fait remarquer divers penseurs contemporains d'horizons très divers, comme René Thom, Hans Jonas, Ernst Mayr, Francisco Varela, Dieter Wandschneider et Paul Davies. S'il est hasardeux de donner une définition de la vie, ce concept exprime une tendance innée présente chez les vivants depuis ses formes les plus élémentaires jusqu'aux plus complexes, depuis l'unicellulaire jusqu'aux sociétés humaines, et peut aussi s'appliquer aux autres formes de vies possibles, même celles qui ne seraient pas ou plus soumises à la sélection naturelle, même aussi les spirituelles, comme l'homme. La dynamique du vivant, non seulement avec son évolution darwinienne, mais surtout avec une évolution quadri dimensionnelle

16 Ce concept de tabula rasa ou tabula in qua nihil scriptum est (tablette vide où rien n'a encore été écrit, permet d'écrire n'importe quoi), est souvent repris depuis Aristote (III de Anima) : cf. par exemple $S$. Th. III ${ }^{a}$, 9, 1, c. ; C. G., 2, 49. 
(Jablonka and Lamb 2006), aspire à produire plus d'autonomie tant aux niveaux systémiques qu'au niveau individuel. Cette omniprésence de l'autonomie sous ses formes diverses, laisse entrevoir une trame analogique entre tous les vivants. L'autonomie est cependant toujours associée chez les créatures vivantes à des dépendances. Certains penseurs comme Hans Jonas et Dieter Wandschneider n'ont pas hésité à établir une analogie profonde entre l'autonomie des vivants et son degré suprême dans la liberté humaine, qui font écho à mes oreilles à la théorie scholastique de la participation, avec l'Analogum Supremum de la perfection vivante dans l'être divin.

Terminons par une remarque qui nous intéresse de près. Depuis l'apparition de l'homme, diverses espèces domestiques ont eu une évolution contrôlée, au point de pouvoir affirmer que ces espèces ne sont pas proprement naturelles : les races bovines Holstein hyper-productives, sélectionnées artificiellement depuis 2000 ans, et la «tulipomanie» du 19e siècle montraient déjà l'intérêt de produire des espèces artificielles, comme le roman «La tulipe noire» (1850) d'Alexandre Dumas l'illustre. L'homme n'a cessé d'avancer dans ce sens et ces succès du contrôle humain sur la nature ne sont pas sans risques. Le danger existe de restreindre et contrôler l'autonomie pour des intérêts à courts termes. L'intervention humaine croissante avec ses sélections artificielles, ses manipulations génétiques et sa technologie est en passe d'ouvrir d'autres types de vie. La gestion de l'autonomie et de la liberté humaine constitue un défi pour la gestion de la nature, comme Jonas a tenté de mettre en avant, sans tomber dans un catastrophisme pessimiste.

\section{Bibliographie}

Aristote, De anima.

Augustin, Confessions.

Dalleur, P. 2008. “Finalité, téléologie et progrès en Biologie.” Acta Philosophica, 17(1): 43-86.

Dalleur, P. 2006. "Fécondité de la notion de «bord» des formes vivantes chez Thom.” Revue philosophique de Louvain, 104 (2): 312-346.

Davies, P. 2003. The Origin of Life. London: Penguin Books. 
Heylighen, F. 2008. “Complexity and Self-organization.” In Encyclopedia of Library and Information Sciences, edited by M. J. Bates and M. N. Maack, 1215-1224. New York: Taylor and Francis.

Jablonka, E., and Lamb M.J. 2006. Evolution in Four Dimensions. Genetic, Epigenetic, Behavioral, and Symbolic Variation in the History of Life. Boston, MA: MIT press. Jonas, H. 2001. The Phenomenon of Life: Toward a Philosophical Biology. Noyes St. Evanston, IL: Northwestern University Press.

Monod, J. 1973. Le Hasard et la Nécessité. Paris: Seuil.

Morin, E. 1980. La Méthode. 2 - La Vie de la Vie. Paris: Seuil.

Fortin, R. 2005. Comprendre la complexité: introduction à La Méthode d'Edgar Morin. Québec: Presses Université Laval.

Pichot, A. 1993. Histoire de la notion de vie. Paris: Gallimard.

Thom, R. 1980. Modèles mathématiques de la morphogenèse. Paris: Christian Bourgeois.

Schrödinger, E. 1944. What is Life? Cambridge: Cambridge University Press.

Varela, F. J. 1997. “Patterns of life: Intertwining identity and cognition.” Brain and Cognition 34(1): 72-87.

Wandschneider, D. 2005. "On the Problem of Direction and Goal in Biological Evolution.” In Darwinism and Philosophy, edited by V. Hösle and C. Illies, 196-215. Notre-Dame, IN: University of Notre-Dame. 